\title{
Erratum: Heuristic Role Detection of Visual Elements of Web Pages
}

\author{
M. Elgin Akpınar ${ }^{1}$ and Yeliz Yeşilada ${ }^{2}$ \\ ${ }^{1}$ Middle East Technical University, Ankara, Turkey \\ ${ }^{2}$ Middle East Technical University Northern Cyprus Campus, \\ Kalkanl, Güzelyurt, Mersin 10, Turkey \\ \{elgin.akpinar, yyeliz\}@metu.edu.tr
}

F. Daniel, P. Dolog, and Q. Li (Eds.): ICWE 2013, LNCS 7977, pp. 123-131, 2013.

(C) Springer-Verlag Berlin Heidelberg 2013

\section{DOI 10.1007/978-3-642-39200-9_51}

The following acknowledgment was inadvertently omitted from the paper.

\section{Acknowledgements}

The project is supported by the Scientific and Technological Research Council of Turkey (TÜBİTAK) with the grant number 109E251 (http://emine.ncc.metu.edu.tr/). As such the authors would like to thank TÜBİTAK for their continued support. 